\title{
Control of Earthquake Induced Vibrations in Asymmetric Buildings Using Passive Damping
}

\author{
Rakesh K. Goel, California Polytechnic State University, San Luis Obispo
}

\begin{abstract}
This paper summarizes the results of a recent investigation on the dynamic response of asymmetric-plan buildings with supplemental viscous damping to harmonic ground motion using modal analysis techniques. It is shown that most modal parameters, except modal damping ratios and dynamic amplification factors, are affected very little by the plan-wise distribution of supplemental damping in the practical range of system parameters. The first modal damping ratio increases while the second decreases as CSD moves from right to left of the system plan, and their values increase with larger plan-wise spread of the supplemental damping. Trends for the dynamic amplification factors are reversed, as they are inversely influenced by the damping ratio, i.e., higher the damping lower the dynamic amplification factor. The largest reduction in the flexible edge deformation occurs when damping in the first mode is maximized.
\end{abstract}

\section{Introduction}

A research program has been designed to systematically investigate the seismic behavior of linearly-elastic, one-story, asymmetric-plan systems with supplemental viscous damping devices. First, three additional system parameters were identified: the damping ratio due to supplemental damping devices, $\zeta_{s d}$; the normalized supplemental damping eccentricity, $\bar{e}_{s d}$; and the normalized supplemental damping radius of gyration, $\bar{\rho}_{s d}$. Next, the effects of these parameters on the flexible and stiff edges of asymmetric-plan systems subjected to a selected earthquake ground motion were investigated. It was shown that supplemental damping reduces edge deformations, and that the degree of reduction strongly depends on the plan-wise distribution of the supplemental damping. Results of this research were reported earlier (Goel, 1997). 
Subsequent research focused on developing a fundamental understanding of why certain plan-wise distribution of damping lead to higher reduction in edge deformations with specific objectives of developing the necessary theoretical background for modal analysis of asymmetric plan buildings with supplemental viscous damping, and systematically investigating how various modal parameters and deformations are affected by the plan-wise distribution of supplemental damping. This paper summarizes the findings of this research; details are available in a full-length journal publication (Goel, 1999).

Presented first is the theoretical background necessary for modal analysis in the complex domain, followed by description of the system and related parameters. Subsequently, the effects of plan wise distribution of supplemental damping on the modal parameters are investigated. Finally, the effects on modal deformations at the two extreme edges are examined.

\section{Theoretical Background}

For non-proportionally damped systems, equations of motion in the state-space are given as:

$$
\boldsymbol{A} \dot{\boldsymbol{z}}(t)+\boldsymbol{B} \boldsymbol{z}(t)=\boldsymbol{R} \ddot{u}_{g}(t)
$$

where $\boldsymbol{z}(t)=\langle\boldsymbol{u}(t) \quad \dot{\boldsymbol{u}}(t)\rangle^{T}$ is a $2 N \times 1$ vector;

$$
\boldsymbol{A}=\left[\begin{array}{cc}
-\boldsymbol{K} & \boldsymbol{0} \\
\boldsymbol{0} & \boldsymbol{M}
\end{array}\right], \boldsymbol{B}=\left[\begin{array}{ll}
\boldsymbol{0} & \boldsymbol{K} \\
\boldsymbol{K} & \boldsymbol{C}
\end{array}\right] \text {, and } \boldsymbol{R}=\left\{\begin{array}{c}
\boldsymbol{0} \\
-\boldsymbol{M r}
\end{array}\right\}
$$

$\boldsymbol{M}, \boldsymbol{C}$, and $\boldsymbol{K}$ characterize the mass, damping, and stiffness related to the deformations $\boldsymbol{u}(t)$ at various degrees of freedom; $\boldsymbol{r}$ is the influence vector; and $\ddot{u}_{g}(t)$ is the ground acceleration. For a system with $N$ degrees-of-freedom (DOF), $\boldsymbol{A}$ and $\boldsymbol{B}$ are $2 N \times 2 N$ matrices; $\boldsymbol{M}, \boldsymbol{C}$, and $\boldsymbol{K}$ are $N \times N$ matrices; and $\boldsymbol{u}(t)$ and $\boldsymbol{r}$ are $N \times 1$ vectors.

Solving the quadratic eigenvalue problem, obtained from the state-space formulation,

$$
(B+\lambda A) \Phi=0
$$

gives $2 N$ complex-valued eigenvalues $\lambda_{n}$ and eigenvectors $\boldsymbol{\Phi}_{n}$. The complex eigenvalues $\lambda_{n}$ appear in complex conjugate pairs in the form of

$$
\lambda_{n}=-\zeta_{n} \omega_{n}-j \omega_{n} \sqrt{1-\zeta_{n}^{2}} \text { and } \lambda_{n}^{*}=-\zeta_{n} \omega_{n}+j \omega_{n} \sqrt{1-\zeta_{n}^{2}}
$$


in which $\omega_{n}$ and $\zeta_{n}$ are the apparent natural vibration frequency and apparent modal damping ratio, respectively, associated with the $\mathrm{n}^{\text {th }}$ modal pair. Eq. (4) may be utilized to obtain the apparent vibration frequencies and apparent modal damping ratios as

$$
\omega_{n}=\sqrt{\operatorname{Re}\left(\lambda_{n}\right)^{2}+\operatorname{Im}\left(\lambda_{n}\right)^{2}} \text { and } \zeta_{n}=\frac{-\operatorname{Re}\left(\lambda_{n}\right)}{\sqrt{\operatorname{Re}\left(\lambda_{n}\right)^{2}+\operatorname{Im}\left(\lambda_{n}\right)^{2}}}
$$

For proportionally damped systems, $2 N$ complex-valued eigenvalues $\lambda_{n}$ and eigenvectors $\boldsymbol{\Phi}_{n}$ may easily be transformed to $\mathrm{N}$ real-valued frequencies and mode shapes. For non-proportionally damped systems the mode shapes would always be complex valued and occur in complex conjugate pairs; the real-valued apparent frequencies, however, may be computed from Eq. (5).

\section{Steady-State Response to Harmonic Ground Motion}

Let $z(t)$ be the steady-state response of the system due to harmonic ground acceleration defined by

$\ddot{u}_{g}(t)=\ddot{u}_{g o} e^{j \omega t}$

in which $\ddot{u}_{g o}$ is the peak value of the ground acceleration and $\omega$ is the forcing frequency. The response $z(t)$ to the harmonic ground motion can be computed as (Goel, 1999):

$z_{k}(t)=\sum_{n=1}^{N} \hat{z}_{k n}(t)$

with $\hat{z}_{k n}(t)$ defined as

$\hat{z}_{k n}(t)=\left|\Gamma_{n}\right| \times\left|\Phi_{k n}\right| \times \frac{2 R_{d n}}{\omega_{n}} \times C_{k n} \times \ddot{u}_{g o} e^{j\left(\omega t+\theta_{n}^{R}+\theta_{k n}^{C}\right)}$

in which $\left|\Gamma_{n}\right|$ and $\left|\Phi_{k n}\right|$ are magnitudes (or absolute values) of the modal participation factor and the mode shape component, respectively; $R_{d n}$ is the dynamic amplification factor; $C_{k n}$ is the angular constant; $\theta_{n}^{R}$ is the phase angle; and $\theta_{k n}^{C}$ is the angle.

\section{One-Story System and Parameters Considered}

The system considered was the idealized one-story building of Fig. 1 consisting of a rigid deck supported by structural elements (wall, columns, moment-frames, braced-frames, etc.) in each of the two orthogonal directions, and included fluid 
viscous dampers incorporated into the bracing system. The mass properties of the system were assumed to be symmetric about both the $X$-and $Y$-axes whereas the stiffness and the damper properties were considered to be symmetric only about the $X$-axis. The distance between the center of mass (CM) and the center of supplemental damping (CSD) is denoted by the supplemental damping eccentricity, $e_{s d}$, whereas distance between the $\mathrm{CM}$ and the center of rigidity $(\mathrm{CR})$ is defined by the stiffness eccentricities, $e$.
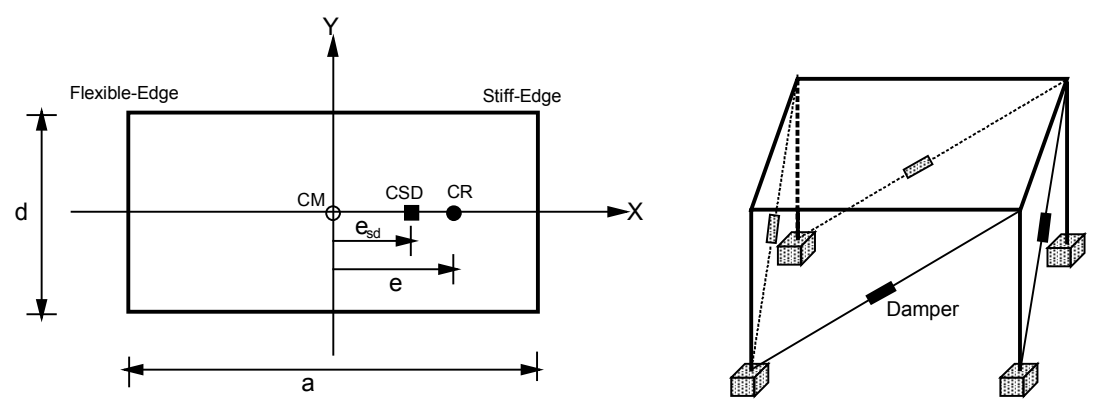

Figure 1. One-story asymmetric plan system with supplemental viscous damping.

The following system parameters were considered in this investigation: $\Omega_{\theta}=1$ to represents systems with strong coupling between lateral and torsional motions in the elastic range; $\bar{e}=0.2$ which implies an eccentricity of $20 \%$ of the plan dimension; 5\% damping ratio in both vibration modes of the system without supplemental damping; and $\zeta_{s d}=10 \%$. The $\bar{e}_{s d}$ was varied between the extreme values of -0.5 to 0.5 . The selected values of $\bar{\rho}_{s d}=0,0.2$, and 0.5 represent low, medium, and large spreads of the supplemental damping about the CSD.

\section{Effects of System Parameters on Modal Properties}

Effects of the plan-wise distribution of supplemental damping on various modal properties - apparent modal periods, apparent damping ratios, mode shape components, modal participation factors, and dynamic amplification factors - were examined. It was found that among all the modal parameters, only apparent damping ratios and dynamic amplification factors are significantly affected by the plan-wise distribution of supplemental damping. Therefore, these modal quantities are discussed next; details for other quantities may be found in Goel (1999).

Damping Ratios. Fig. 2 presents variation of apparent modal damping ratios, $\zeta_{1}$ and $\zeta_{2}$, with $\bar{e}_{s d}$ and $\bar{\rho}_{s d}$. This figure leads to the following conclusions. The apparent modal damping ratios are significantly affected by both $\bar{e}_{s d}$ and $\bar{\rho}_{s d}$. In particular, $\zeta_{1}$ decreases and $\zeta_{2}$ increases as the CSD moves from left to right in the system plan, i.e., $\bar{e}_{s d}$ varies from -0.5 to 0.5 , and both $\zeta_{1}$ and $\zeta_{2}$ become larger as $\bar{\rho}_{s d}$ increases. 
Damping ratios much higher than the damping obtained by evenly distributing the supplemental damping in the system plan, i.e., $\bar{e}_{s d}=0$, are possible. Consider, for example, the damping ratios in systems with $\bar{\rho}_{s d}=0.5$. The apparent value of $\zeta_{1}$ is nearly two-and-a-half times for $\bar{e}_{s d}=-0.5$ compared that for $\bar{e}_{s d}=0$; the two values are $62 \%$ and $25 \%$, respectively. Similarly, the apparent value of $\zeta_{2}$ is more than two times for $\bar{e}_{s d}=0.5$ compared to that for $\bar{e}_{s d}=0$; the two values are $43 \%$ and $19 \%$, respectively.

It is also apparent that damping ratios much higher than those in the corresponding symmetric-plan system are possible with appropriate plan-wise distribution of the supplemental damping. For example, a total of $15 \%$ damping (5\% natural $+10 \%$ supplemental) in the symmetric system may give up to $62 \%$ in the fundamental modal pair of asymmetric-plan system with careful plan-wise distribution of the supplemental damping.

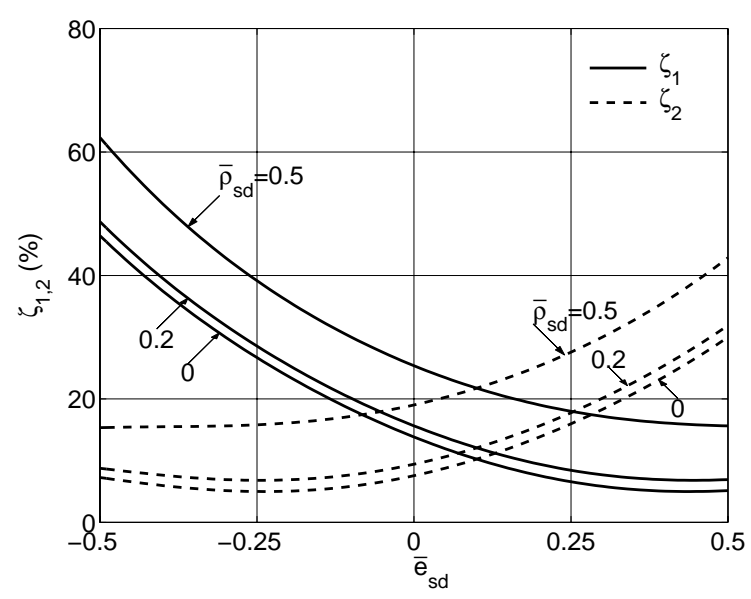

Figure 2. Apparent damping ratios of asymmetric-plan systems with supplemental damping.

The largest possible values of $\zeta_{1}$ and $\zeta_{2}$ do not occur for the same values of $\bar{e}_{s d}$ : $\zeta_{2}$ is nearly at its minimum value when $\zeta_{1}$ reaches its maximum value and vice versa. This indicates that the plan-wise distribution of the supplemental damping, i.e., selection of $\bar{e}_{s d}$, should depend on which of the two modal pairs dominates the response. If the first modal pair dominates, the supplemental damping should be distributed to maximize $\zeta_{1}$ by locating the CSD as far away from the CM, on the side opposite to the $\mathrm{CR}$, as possible, i.e., $\bar{e}_{s d}$ as close to -0.5 as possible. If the second modal pair dominates, then the supplemental damping should be distributed to maximize $\zeta_{2}$ by locating the CSD as far away from the CM, on the same side of the $\mathrm{CR}$, as possible, i.e., $\bar{e}_{s d}$ as close to 0.5 as possible. 
Dynamic Amplification Factor. Figure 3 shows the variation of $R_{d n}$ with the planwise distribution of supplemental damping. Results are presented for $\beta_{1}=1$, i.e., forcing frequency equal to the first apparent modal frequency. Since $R_{d 2}$ is nearly equal to one for $\beta_{2}=\beta_{1} \times T_{2} \div T_{1}<<1, R_{d 2}$ is not included in the figure. The presented results show that $R_{d 1}$ increases as CSD moves from left to right of the system plan, i.e., as $\bar{e}_{s d}$ varies from -0.5 to 0.5 . The degree to which $R_{d 1}$ increases depends on $\bar{\rho}_{s d}$ : larger the value of $\bar{\rho}_{s d}$, smaller the increase. These trends are nearly opposite to the previous observations on $\zeta_{1}$ which decreases as $\bar{e}_{s d}$ varies from -0.5 to 0.5 , and becomes larger as $\bar{\rho}_{s d}$ increases (Fig. 2). This is to be expected because $R_{d n}$ is reduced significantly as damping is increased and vice versa. Although results are not presented here for $\beta_{2}=1$, the results presented in Goel (1999) show that $R_{d 2}$ decreases as CSD moves from left to right of the system plan, i.e., as $\bar{e}_{s d}$ varies from -0.5 to 0.5 and $R_{d 2}$ becomes smaller as value of $\bar{\rho}_{s d}$ increases. The trends for $R_{d 2}$ are related to $\zeta_{2}$ which increases as $\bar{e}_{s d}$ varies from -0.5 to 0.5 , and becomes larger as $\bar{\rho}_{s d}$ increases.

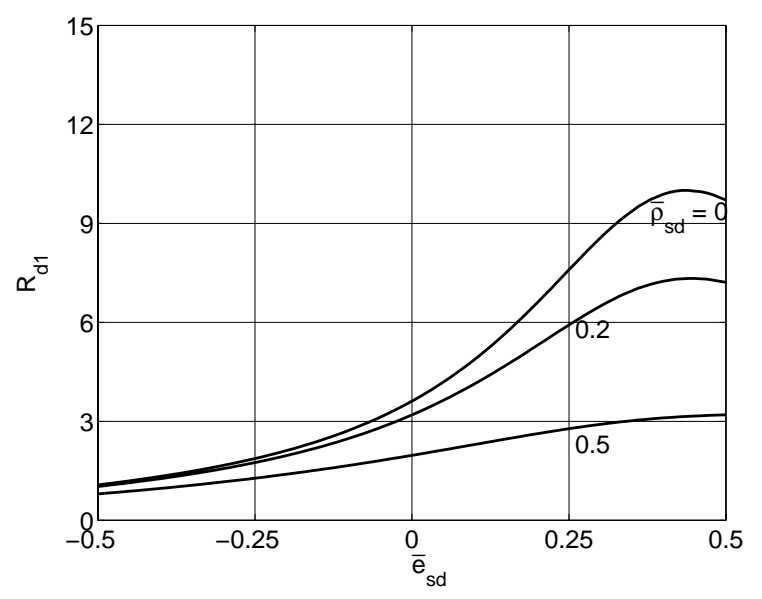

Figure 3. Dynamic response (or amplification factor) for asymmetric-plan systems with supplemental damping; $\beta_{1}=1$.

\section{Effects of System Parameters on Edge Deformations}

Figure 4 presents the deformations at the flexible and stiff edges of the system, $u_{s 1}$ and $u_{f 1}$, due to the first modal pair for $\beta_{1}=1$. As expected, deformations on the flexible edge, $u_{f 1}$, are much larger than those on the stiff edge, $u_{s 1}$. The edge deformations are the smallest for $\bar{e}_{s d}=-0.5$. They increase as the CSD moves from the left to right, i.e., $\bar{e}_{s d}$ varies from -0.5 to 0.5 and reach their maximum value near $\bar{e}_{s d}=0.5$. Although results are not presented for reasons of brevity, the opposite trends may be expected for $\beta_{2}=1$ 
The dependence of the edge deformations on $\bar{e}_{s d}$ as well as $\bar{\rho}_{s d}$ is the largest for $\bar{e}_{s d}>0$. For $\bar{e}_{s d}<0$, especially for values of $\bar{e}_{s d}$ between -0.25 and -0.5 , the edge deformations are affected very little by either $\bar{e}_{s d}$, as indicated by flattening of the curves, or by $\bar{\rho}_{s d}$, as apparent from closeness of the three curves for $\bar{\rho}_{s d}=0,0.2$ and 0.5 .

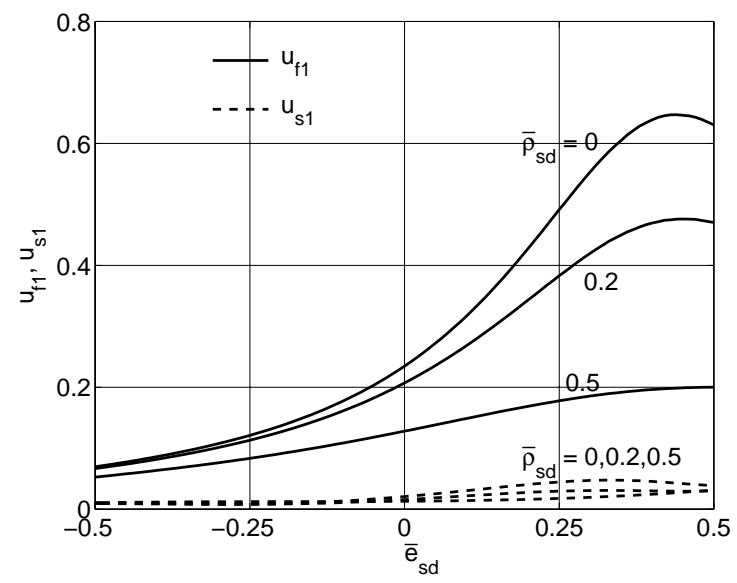

Figure 4. Deformations at the flexible and stiff edge of asymmetric-plan systems with supplemental damping due to first modal pair: $\beta_{1}=1$.

Since one of the major concerns for asymmetric plan buildings is to reduce deformations on the flexible edge, the plan-wise distribution of the supplemental damping should be such that deformation of this edge are reduced the most. The presented results suggest that this objective would be met if the supplemental damping is distributed such the CSD is as far away from the CM, on the side opposite to the CR, as possible, i.e., $\bar{e}_{s d}$ as close to -0.5 as possible, and $\bar{\rho}_{s d}$ is as large as possible. Such a distribution corresponds to maximizing the apparent modal damping in the first mode (Fig. 2). Since value of $\bar{e}_{s d}$ as close to -0.5 as possible and largest value of $\bar{\rho}_{s d}$ can not be physically obtained simultaneously, it may be sufficient to distribute supplemental damping such that $\bar{e}_{s d}$ is equal to in magnitude but opposite in algebraic sign to the structural eccentricity. This distribution leads to near optimal reduction in the flexible edge deformation; additional reductions, although possible, are small because of the low sensitivity of the deformation in this range of system parameters.

\section{Conclusions}

It is demonstrated that most modal parameters, except dynamic amplification factor, are affected very little by the plan-wise distribution of supplemental damping. This is especially true for practical range of the system parameters. Dynamic amplification factor (DAF) is significantly affected by the plan-wise distribution of supplemental damping. If the forcing frequency is close to the first apparent 
frequency, i.e., $\beta_{1}=1, R_{d 1}$ increases as CSD moves from left to right of the system plan, i.e., as $\bar{e}_{s d}$ varies from -0.5 to 0.5 . If the forcing frequency is close to the second apparent frequency, i.e., $\beta_{2}=1, R_{d 2}$ increases as CSD moves from right to left of the system plan, i.e., as $\bar{e}_{s d}$ varies from 0.5 to -0.5 . DAF becomes smaller with larger the value of $\bar{\rho}_{s d}$. These trends for DAF are directly related to the apparent modal damping ratios, $\zeta_{1}$ and $\zeta_{2}$. $\zeta_{1}$ increases and $\zeta_{2}$ decreases as CSD moves from right to left of the system plan, i.e., as $\bar{e}_{s d}$ varies from 0.5 to -0.5 . Both $\zeta_{1}$ and $\zeta_{2}$ become larger as $\bar{\rho}_{s d}$ increases.

For obtaining the largest reduction in the flexible edge deformation, which is generally the most critical edge, the supplemental damping should be distributed such the CSD is as far away from the CM, on the side opposite to the CR, as possible and $\bar{\rho}_{s d}$ is as large as possible. Since both these criteria can not be physically satisfied simultaneously, it may be sufficient to distribute supplemental damping such that $\bar{e}_{s d}$ is equal to in magnitude but opposite in algebraic sign to the structural eccentricity.

\section{Acknowledgements}

This research investigation is funded by the National Science Foundation under Grant CMS-9812414. This financial support is gratefully acknowledged.

\section{References}

Goel, R. K. (1997). "Effects of Supplemental Viscous Damping on Seismic Response of Asymmetric-Plan Systems" Earthquake Engineering and Structural Dynamics, Vol. 27, pp. 125-141.

Goel, R. K. (1999). "Seismic Behavior of Asymmetric Buildings with Supplemental Damping," Accepted for Publication, Earthquake Engineering and Structural Dynamics, September. 